ARTICLE

Received 23 Jun 2013 | Accepted 1 Nov 2013 | Published 10 Dec 2013

DOI: $10.1038 /$ ncomms3854

OPEN

\title{
Influenza $A(H 7 N 9)$ virus gains neuraminidase inhibitor resistance without loss of in vivo virulence or transmissibility
}

Rong Hai ${ }^{1, \star}$, Mirco Schmolke ${ }^{1,2, \star}$, Victor H. Leyva-Grado ${ }^{1}$, Rajagowthamee R. Thangavel ${ }^{1}$, Irina Margine ${ }^{1}$, Eric L. Jaffe ${ }^{1}$, Florian Krammer ${ }^{1}$, Alicia Solórzano ${ }^{3}$, Adolfo García-Sastre ${ }^{1,2,4}$, Peter Palese ${ }^{1,4}$

\& Nicole M. Bouvier ${ }^{1,4}$

Without baseline human immunity to the emergent avian influenza $A(H 7 N 9)$ virus, neuraminidase inhibitors are vital for controlling viral replication in severe infections. An amino acid change in the viral neuraminidase associated with drug resistance, NA-R292K (N2 numbering), has been found in some H7N9 clinical isolates. Here we assess the impact of the NA-R292K substitution on antiviral sensitivity and viral replication, pathogenicity and transmissibility of H7N9 viruses. Our data indicate that an H7N9 isolate encoding the NAR292K substitution is highly resistant to oseltamivir and peramivir and partially resistant to zanamivir. Furthermore, H7N9 reassortants with and without the resistance mutation demonstrate comparable viral replication in primary human respiratory cells, virulence in mice and transmissibility in guinea pigs. Thus, in stark contrast to oseltamivir-resistant seasonal influenza $A(H 3 N 2)$ viruses, H7N9 virus replication and pathogenicity in these models are not substantially altered by the acquisition of high-level oseltamivir resistance due to the NA-R292K mutation.

\footnotetext{
${ }^{1}$ Department of Microbiology, Icahn School of Medicine at Mount Sinai, One Gustave L Levy Place, Box 1124, New York, New York 10029, USA. ${ }^{2}$ Global Health and Emerging Pathogens Institute, Icahn School of Medicine at Mount Sinai, One Gustave L Levy Place, Box 1124, New York, New York 10029, USA. ${ }^{3}$ Public Health Research Institute and Regional Biocontainment Laboratory, New Jersey Medical School, RUTGERS, The State University of New Jersey, 225 Warren Street, Newark, New Jersey 07103, USA. ${ }^{2}$ Division of Infectious Diseases, Department of Medicine, Icahn School of Medicine at Mount Sinai, One Gustave L Levy Place, Box 1124, New York, New York 10029, USA. * These authors contributed equally to this work. Correspondence and requests for materials should be addressed to N.M.B. (email: nicole.bouvier@mssm.edu).
} 
S ince the first documented direct transmission of highly pathogenic avian influenza $\mathrm{A}(\mathrm{H} 5 \mathrm{~N} 1)$ virus from poultry to humans in the late 1990s, zoonotic influenza A virus infections have been considered to pose a threat to public health. Though all clade 1 and many clade 2 avian H5N1 viruses are resistant to amantadine, the development of neuraminidase (NA) inhibitor resistance in human $\mathrm{H} 5 \mathrm{~N} 1$ infection has fortunately been rare, and only three oseltamivir-resistant strains have been described ${ }^{1-3}$

On 31 March 2013, Chinese public health authorities reported laboratory confirmation of avian-origin H7N9 influenza virus infections in three people in the greater Shanghai area ${ }^{4}$. Since then, 135 total laboratory-confirmed cases have been reported, resulting in 44 fatalities ${ }^{5}$. Although the source of these infections has not conclusively been confirmed, direct exposure to poultry has been reported in the majority of cases. In consequence, public health authorities closed down poultry markets on 6 April 2013, thus limiting the numbers of new infections ${ }^{4}$. Some live poultry markets began reopening in June under new, stricter infection control standards (http://www.globaltimes.cn/content/ 790164.shtml -.Ulw_YII1fo8); however, the possibility of avianto-human transmission still exists.

Annual vaccination is still the most effective means of reducing influenza-associated illness and death. However, in the case of a newly emerging influenza virus strain, there is a gap between the epidemic onset and the availability of subtype-specific vaccine supplies, as was seen in the swine-origin H1N1 pandemic in 2009 (ref. 6). The prophylactic use of antivirals, particularly in persons at high risk for complicated influenza, can at least partially bridge this gap. Moreover, the timely administration of antivirals to patients severely ill with seasonal, pandemic and avian influenza has been associated with improved clinical outcomes ${ }^{7}$. Two classes of antivirals are available for treatment of acute influenza A virus infection: amantadines and NA inhibitors. For both classes, resistant virus mutants have been described; in fact, treatment of influenza disease currently relies solely on NA inhibitors, because the majority of influenza A and all influenza B isolates infecting humans are resistant to the action of amantadine and rimantadine ${ }^{7}$.

The influenza virus NA is a membrane-expressed, glycosylated enzyme with sialidase function, cleaving cell-surface sialic acid receptors to which the haemagglutinin (HA) glycoprotein binds. Its enzymatic activity is critical for the efficient egress of mature viral particles from sialylated host cells, and for the destruction of respiratory tract decoy receptors that impede infection by competing for HA binding 8 . NA inhibitors are sialic acid analogues that block the enzymatic active site and prevent its sialidase activity ${ }^{9}$, and numerous subtype-specific mutations that confer reduced susceptibility to this drug class have been described $^{10}$. Among them, a single nucleotide change in the NA gene can generate resistance to NA inhibitors via an arginine-tolysine amino acid substitution in the enzymatic active site (R292K in N2 numbering; R294K in N9 numbering ${ }^{11}$ ), and this NA-R292K mutation has been reported in patients infected with H7N9 influenza A viruses and treated with NA inhibitors ${ }^{11,12}$. However, resistance mutations usually result in a loss in viral fitness, which may be restored by additional compensatory changes in the viral genome $e^{13-15}$. Here we investigate whether the R292K-encoding NA of a recent H7N9 clinical isolate confers NA inhibitor resistance and whether it affects the replication or pathogenicity of this strain in primary human tracheobronchial epithelial (hTBE) cell culture and in a mouse virulence model. We also explore the replicative ability of oseltamivir-sensitive and -resistant H7N9 viruses in the lungs of mice treated with oseltamivir or zanamivir, as well as the respiratory droplet transmissibility of these viruses in the guinea pig model.
Our results demonstrate that the acquisition of high-level oseltamivir resistance, via the NA-R292K mutation, can significantly impair the sialidase activity of the H7N9 NA without compromising viral replication, virulence or transmissibility in these experimental models. We hypothesize that maintenance of virus fitness is enabled, at least in part, by concurrent, compensatory changes in the expression of HA. The development of oseltamivir resistance, unaccompanied by demonstrable losses of replicative ability in vitro or of virulence or transmissibility in vivo, underscores the multigenic and sometimes unpredictable nature of fitness in influenza A viruses.

\section{Results}

Susceptibility to NA inhibitors. Of the first three influenza $\mathrm{A}(\mathrm{H} 7 \mathrm{~N} 9)$ virus isolates collected from Chinese patients (Shanghai/1, Shanghai/2 and Anhui/1), one, Shanghai/1, was found to encode the NA mutation R292K (in N2 numbering, corresponding to residue 294 in the N9 NA). In group 2 NAs, including $\mathrm{N} 2$ and $\mathrm{N} 9$, this amino-acid substitution has been shown to contribute to decreased susceptibility to NA inhibitors $^{16-19}$. Thus, we hypothesized that the Shanghai/1-NA would have some degree of inherent resistance to this drug class. To evaluate this prediction experimentally, we generated recombinant A/Puerto Rico/8/1934(H1N1)-based 7:1 reassortant viruses expressing either the Shanghai/1-NA (rPR/8:SH/1-NA) or the Anhui/1-NA (rPR/8:AH/1-NA) by reverse genetics. Using the fluorogenic substrate MUNANA, we calculated the median inhibitory concentration $\left(\mathrm{IC}_{50}\right)$ at which the NA inhibitors oseltamivir carboxylate, peramivir and zanamivir inhibited 50\% of maximal enzymatic activity. Compared with the Anhui/1-NA, the Shanghai/1-NA was highly resistant to inhibition by oseltamivir (with a $>4,600$-fold higher $\mathrm{IC}_{50}$ ), moderately resistant to peramivir (563-fold higher) and mildly resistant to zanamivir (11-fold higher) (Table 1).

NA enzymatic activity and substrate affinity. To place these isolates in the context of what is known about the enzymatic activity of oseltamivir-sensitive and -resistant group 2 NAs, we performed MUNANA-based kinetic assays on the 7:1 PR/8 reassortant viruses, normalized to equivalent NA content, to derive the Michaelis-Menten parameters $V_{\max }$ and $K_{\mathrm{m}}$ (Table 2). Compared with the oseltamivir-sensitive Anhui/1-NA, the oseltamivir-resistant Shanghai/1-NA demonstrated an $\sim 80 \%$ reduction in both maximal enzymatic activity $\left(V_{\max }\right)$ and affinity for the MUNANA substrate $\left(1 / K_{\mathrm{m}}\right)$. When $7: 1$ reassortant viruses, diluted to equivalent NA content, were assessed for haemagglutination activity, the haemagglutination unit titre measured for the $\mathrm{rPR} / 8: \mathrm{AH} / 1-\mathrm{NA}$ virus was two- to fourfold higher than that obtained for $\mathrm{rPR} / 8: \mathrm{SH} / 1-\mathrm{NA}$. Thus, relative to oseltamivir-sensitive rPR/8:AH/1-NA, oseltamivir-resistant $\mathrm{rPR} /$ 8:SH/1-NA displays comparable in vitro reductions in HA receptor binding activity and NA enzymatic function.

Table 1 | Resistance of the Shanghai/1-NA to NA inhibitors.

\begin{tabular}{|c|c|c|c|}
\hline \multirow{2}{*}{$\begin{array}{l}\text { NA } \\
\text { Inhibitor }\end{array}$} & \multicolumn{2}{|c|}{ Mean IC $50(95 \% \mathrm{Cl})(\mathrm{nM})$} & \multirow{2}{*}{$\begin{array}{c}\text { Relative } \\
\text { resistance } \\
\text { (SH/AH) }\end{array}$} \\
\hline & AH/1-NA (R292) & SH/1-NA (R292K) & \\
\hline Oseltamivir & $1.87(1.65-2.11)$ & $8,620(6,590-11,300)$ & 4,610 \\
\hline Peramivir & $0.339(0.259-0.443)$ & $191(150-242)$ & 563 \\
\hline Zanamivir & $3.75(3.26-4.30)$ & $40.1(30.1-53.4)$ & 11 \\
\hline
\end{tabular}




\begin{tabular}{|c|c|c|c|}
\hline & $\begin{array}{c}\text { PR/8: AH/1- } \\
\text { NA (R292) }\end{array}$ & $\begin{array}{c}\text { PR/8: SH/1-NA } \\
\text { (R292K) }\end{array}$ & $\begin{array}{l}\text { Fold difference } \\
\text { (SH/AH) }\end{array}$ \\
\hline $\begin{array}{l}\text { Geometric mean } \\
H A U \pm \text { s.e. }\end{array}$ & $724 \pm 1.4$ & $256 \pm 1.0$ & 0.35 \\
\hline $\begin{array}{l}\text { Mean } K_{m} \pm \text { s.e. } \\
(\mu \mathrm{M})\end{array}$ & $464 \pm 21$ & $2,550 \pm 401$ & \\
\hline $\begin{array}{l}\left(\text { Mean } K_{\mathrm{m}}\right)^{-1} \\
\left(\mathrm{nM}^{-1}\right)\end{array}$ & 2.16 & 0.392 & 0.18 \\
\hline $\begin{array}{l}\text { Mean } V_{\max } \pm \text { s.e. } \\
\left(\text { RFU }^{-1}\right)\end{array}$ & $26.1 \pm 0.57$ & $5.38 \pm 0.66$ & 0.21 \\
\hline
\end{tabular}

Virus replication kinetics in hTBE cells. To investigate the impact of oseltamivir resistance on the replication of H7N9 viruses, we replaced the oseltamivir-resistant NA of Shanghai/1 with the oseltamivir-sensitive NA of Anhui/1 (rSH/1: AH/1-NA) and compared this virus to recombinant wild-type Shanghai/1 $(\mathrm{rSH} / 1)$.

Efficient replication in the upper respiratory tract is thought to be essential for viral transmission among humans. To assess the effect of oseltamivir resistance on the replication of H7N9 viruses in a relevant primary cell culture model, we infected differentiated hTBE cells with either $\mathrm{rSH} / 1$ or $\mathrm{rSH} / 1: \mathrm{AH} / 1-\mathrm{NA}$ at a multiplicity of infection (MOI) of 0.01 , incubated the infected cells at $33^{\circ} \mathrm{C}$ or $37^{\circ} \mathrm{C}$, and determined virus titres at $8,24,48$ and $72 \mathrm{~h}$ post infection (Fig. 1). At each temperature, $\mathrm{rSH} / 1$ and $\mathrm{rSH} / 1: \mathrm{AH} / 1$ NA showed similar replication kinetics and peak titres, even though both viruses replicated faster at $37^{\circ} \mathrm{C}$ than at $33^{\circ} \mathrm{C}$. Thus, our findings indicate that the oseltamivir-resistant NA encoded by A/Shanghai/1 does not compromise the replication of this virus in primary human upper respiratory tract cells.

Virus replication in mice. Next, we examined the impact of oseltamivir resistance on viral replication in vivo. BALB/c mice were infected with 100 plaque-forming units (PFU) of $\mathrm{rSH} / 1$ or $\mathrm{rSH} / 1: \mathrm{AH} / 1-\mathrm{NA}$. On day 2, 4 and 6 post infection, virus titres in the lungs of mice were determined (Fig. 2a). We observed no significant difference in the replication kinetics of the oseltamivirresistant isolate $(\mathrm{rSH} / 1)$ as compared with the oseltamivir-sensitive one (rSH/1:AH/-NA). We also assayed for virus replication in spleen and intestinal tissue; however, no virus was recovered from these extra-pulmonary tissues at any time point (Fig. 2a). These findings indicate that the oseltamivir-resistant NA does not compromise virus replication in the lung or alter its tissue tropism in the mouse model.

Virulence in mice. The attainment of oseltamivir resistance can result in viral attenuation ${ }^{3,19,20}$. To investigate a potential influence of oseltamivir resistance on virulence of $\mathrm{H} 7 \mathrm{~N} 9$ viruses in vivo, we determined the median mouse lethal dose $\left(\mathrm{mLD}_{50}\right)$ of $\mathrm{rSH} / 1$ and $\mathrm{rSH} / 1: \mathrm{AH} / 1-\mathrm{NA}$. We infected $\mathrm{BALB} / \mathrm{c}$ mice intranasally with 10 -fold serial dilutions of each virus (from $10^{4}$ to $10^{1}$ PFU per mouse), and weight and health status were monitored daily for 14 days post-infection. Mice infected with both viruses demonstrated comparable weight loss patterns (Fig. 2b-e) and similar onset of clinical signs of disease, including ruffled fur, hunching and lethargy. The Kaplan-Meier survival curves comparing the two viruses at each inoculating dose were compared by the log-rank test (Fig. $2 \mathrm{f}-\mathrm{i}$ and Table 3 ). The median time to death in the $10^{3}$ and $10^{2} \mathrm{PFU}$ dose groups

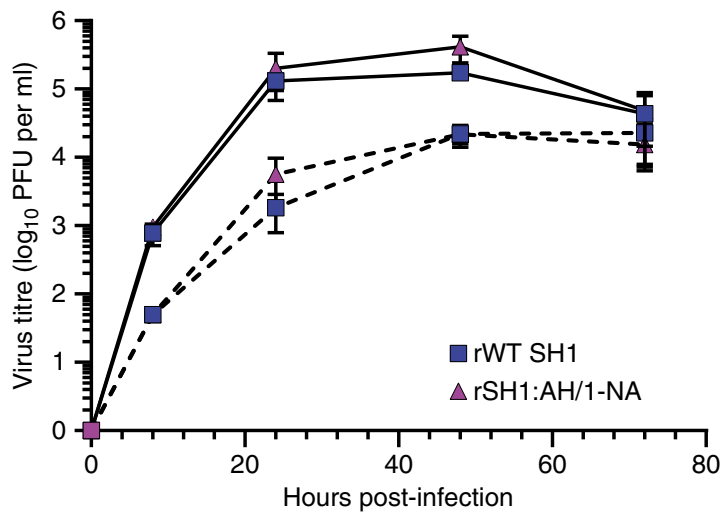

Figure 1 | Oseltamivir resistance does not affect viral replication in human tracheobronchial epithelial cells. Differentiated human tracheobronchial epithelial cells were infected with $\mathrm{rSH} / 1$ (squares) or $\mathrm{rSH} / 1: \mathrm{AH} / 1-\mathrm{NA}$ (triangles) viruses, at an $\mathrm{MOI}$ of 0.01 , at either $33^{\circ} \mathrm{C}$ (dashed line) or $37^{\circ} \mathrm{C}$ (solid line). At $8,24,48$ and $72 \mathrm{~h}$ post infection, cells were washed with PBS/BSA and the concentration of virus in each wash sample was determined by standard plaque assay on MDCK cells. The hTBE growth curves were performed in triplicate. Error bars represent s.d.

were slightly longer for $\mathrm{rSH} / 1: \mathrm{AN} / 1-\mathrm{NA}$ than for $\mathrm{rSH} / 1$ ( $P=0.0495$ and $P=0.0442$, respectively). However, for the oseltamivir-sensitive virus $\mathrm{rSH} / 1: \mathrm{AH} / 1-\mathrm{NA}$ we determined an $\mathrm{mLD}_{50}$ of $42 \mathrm{PFU}$ (95\%CI, 10-170 PFU) and for the oseltamivirresistant virus $\mathrm{rSH} / 1,32 \mathrm{PFU}$ (95\%CI, 8.9-110 PFU), which were not significantly different.

Viral susceptibility to oseltamivir and zanamivir in mice. We further evaluated the in vivo replication of $\mathrm{rSH} / 1$ and $\mathrm{rSH} / 1$ : $\mathrm{AH} / 1-\mathrm{NA}$ in the lungs of mice treated with the NA inhibitors zanamivir and oseltamivir. In the zanamivir treatment group, viral lung titres of both viruses were significantly reduced compared with mice treated with vehicle only (Fig. 3a). Virus titres of $\mathrm{rSH} / 1$ and $\mathrm{rSH} / 1: \mathrm{AH} / 1-\mathrm{NA}$ were comparably reduced in zanamivir-treated mice, consistent with the relatively minor 11 -fold difference in their in vitro susceptibilities to zanamivir. In contrast, we observed a significant, 1 log reduction in viral lung titres of the oseltamivir-sensitive $\mathrm{rSH} / 1$ :AH/1-NA virus in mice treated with oral oseltamivir, whereas oseltamivir treatment had no effect on lung titres in mice infected with the oseltamivir-resistant $\mathrm{rSH} /$ 1 virus (Fig. 3b). These results accord with our in vitro observation of the $>4,600$-fold increase in oseltamivir resistance conferred by the Shanghai/1-NA (Table 1).

Airborne respiratory transmission in guinea pigs. Next, we investigated the impact of oseltamivir resistance on airborne respiratory transmission of $\mathrm{H} 7 \mathrm{~N} 9$ viruses in the guinea pig model. Four guinea pigs were inoculated with $\mathrm{rSH} / 1 \mathrm{AH} / 1-\mathrm{NA}$ and four with $\mathrm{rSH} / 1$. At 1 day post-inoculation, eight naive guinea pigs were exposed to these infected guinea pigs, in cages that precluded direct contact between animals. Transmission events were detected by isolation of infectious influenza virus in the nasal lavage fluid of exposed guinea pigs and confirmed by assessing for H7N9 virus-specific seroconversion (Table 4). The inoculated guinea pigs shed both viruses to similar peak titres with similar timing $\left(10^{5}-10^{6} \mathrm{PFU} \mathrm{ml}^{-1}\right.$ on day 2 post-inoculation). However, these peak nasal lavage virus titres were, on average, $\sim 1$ log lower than those typically observed in guinea pigs inoculated with $\mathrm{H} 3 \mathrm{~N} 2$ and H1N1 human influenza $\mathrm{A}$ isolates ${ }^{14,20,21}$. 
Transmission events were observed in one of four guinea pigs exposed to the oseltamivir-sensitive reassortant $\mathrm{rSH} / 1$ :AN/1-NA and in two of four guinea pigs exposed to the oseltamivir-resistant recombinant $\mathrm{rSH} / 1$, without significant difference in transmission efficiency $(P=1.00)$. Both of the guinea pigs infected by airborne transmission of $\mathrm{rSH} / 1$ seroconverted; however, the one guinea pig infected by transmission of $\mathrm{rSH} / 1$ :AN/1-NA died of anesthesia complications on day 4 post-inoculation, and thus seroconversion could not be assessed (Table 4). Nevertheless, our data suggest that, although airborne transmission of $\mathrm{H} 7 \mathrm{~N} 9$ viruses in guinea pigs is inefficient overall, the acquisition of oseltamivir resistance does not further impair transmissibility. In conclusion, we have shown that the Shanghai/1-NA gene confers high-level oseltamivir resistance in vitro, but the gain of oseltamivir resistance does not attenuate virulence in mice, transmission among guinea pigs or replicative ability in either species.
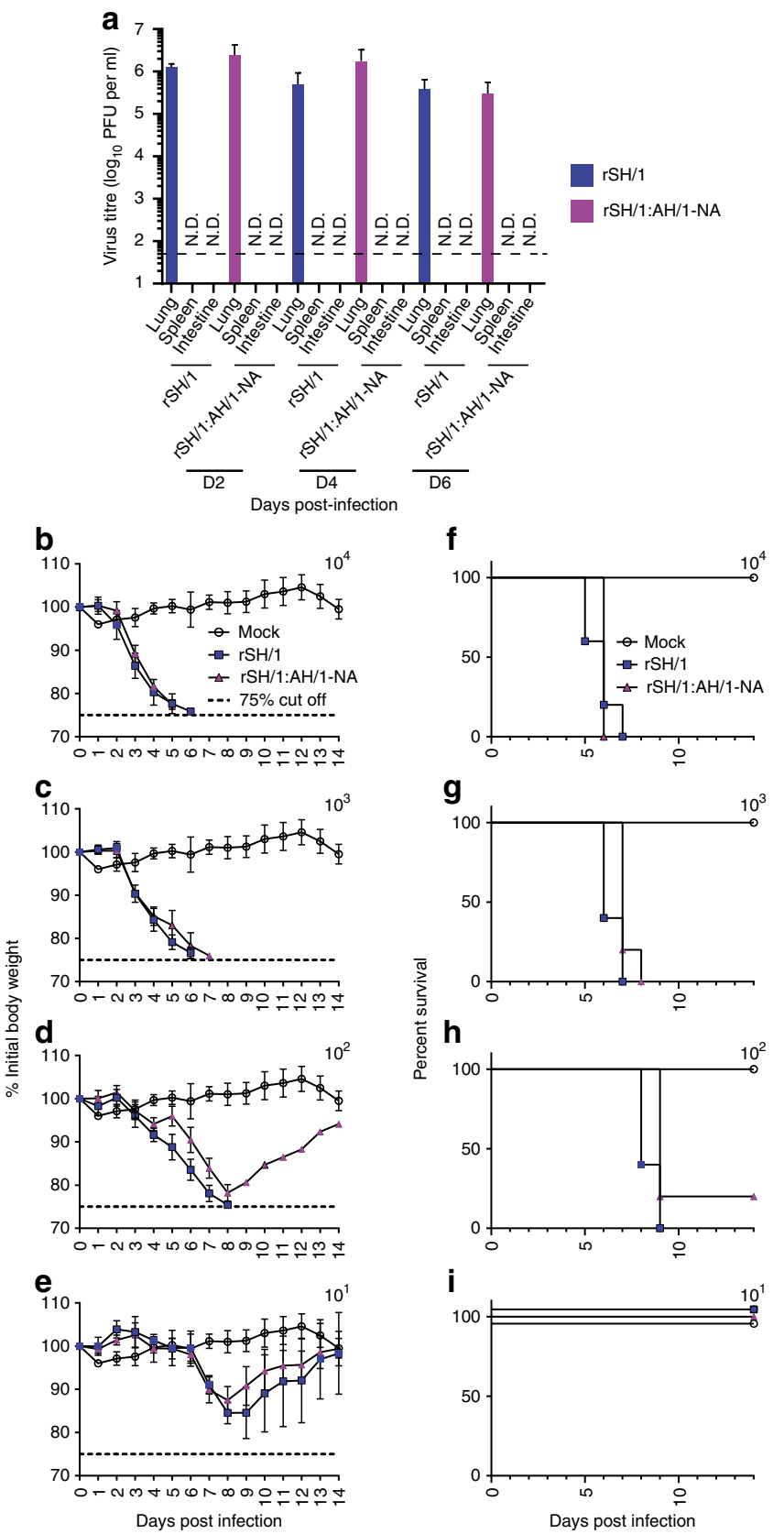

\section{Discussion}

Severe clinical outcomes have been observed in H7N9-infected patients with delayed or ineffective viral clearance; in some cases, identification of the R292K NA inhibitor resistance mutation has been associated with persistent viral replication despite oseltamivir treatment ${ }^{12}$. We thus investigated the susceptibility of two representative H7N9 NAs to inhibition by three clinically available NA inhibitors, as well as the effect of oseltamivir resistance on the in vitro and in vivo replicative capacity and pathogenicity of H7N9 viruses.

The arginine at position 292 (in $\mathrm{N} 2$ numbering, corresponding to N9 R294) is one of seven residues highly conserved among all known viral and bacterial sialidases ${ }^{9}$. It is critical for interaction with sialic acid ${ }^{9}$ and with the sialic acid analogues oseltamivir, peramivir and zanamivir ${ }^{18}$ at the enzyme's active site. Previous studies with the N2 NA have indicated that the NA-R292K mutation not only enhances resistance to the inhibitory action of oseltamivir (and, to a lesser extent, that of peramivir and zanamivir) but also significantly impairs NA activity and virus replicative ability in vitro and in vivo ${ }^{18,19,22,23}$. Among the H7N9 NAs assessed to date, those encoding the NA-R292K substitution, like Shanghai/1, have demonstrated high-level oseltamivir resistance, mid-level peramivir resistance and low-level zanamivir resistance ${ }^{24-26}$. However, in contrast to $\mathrm{H} 3 \mathrm{~N} 2$ viruses, oseltamivir resistance appears not to compromise H7N9 replication in human respiratory tract cells or in mouse lungs ${ }^{25,27,28}$. Recently, the oseltamivir-sensitive H7N9 clinical isolate Anhui/1 has been shown to transmit, albeit inefficiently, among ferrets ${ }^{25,27-30}$, as does the contemporaneous H7N9 isolate Shanghai/1 (refs 27,28). Virus stocks grown from the Shanghai/1 clinical isolate have been found to contain a mixed population of oseltamivir-sensitive (NA-R292) and -resistant (NA-R292K) virus quasi-species, and recent studies have revealed that, in Shanghai/1, a predominantly oseltamivir-sensitive virus population can mask the presence of NA-R292K in a minority variant ${ }^{25,26}$. Thus, the similar virulence and transmission phenotypes shown by Shanghai/1 and Anhui/1 may reflect that the Shanghai/1 clinical isolate comprises a relatively small proportion of oseltamivirresistant quasi-species. To our knowledge, it has yet to be demonstrated that the transmission of Shanghai/1 in ferrets can be

Figure 2 | Oseltamivir resistance in a recombinantly derived H7N9 virus does not modulate viral pathogenicity in mice. Female BALB/C mice (6-8-week-old) were infected intranasally with various amounts of $\mathrm{rSH} / 1$ (squares) or $\mathrm{rSH} / 1: \mathrm{AH} / 1-\mathrm{NA}$ (triangles) viruses. (a) Viral replication in lung, spleen and intestine. Mice $(n=9)$ were infected intranasally with 100 $\mathrm{PFU}$ of $\mathrm{rSH} / 1$ (squares) or $\mathrm{rSH} / 1: \mathrm{AH} / 1-\mathrm{NA}$ (triangles) viruses. On days 2, 4 and 6 post infection, three mice per group were euthanized. The presence of virus in lung, spleen and intestinal tissues was determined by standard plaque assay on MDCK cells. Dashed line indicates the limit of detection $\left(100 \mathrm{PFU} \mathrm{ml}{ }^{-1}\right) . \mathrm{ND}=$ not detected. (b-e) Weight loss of mice after infection with $\mathrm{rSH} / 1$ (squares) or $\mathrm{rSH} / 1: \mathrm{AH} / 1-\mathrm{NA}$ (triangles) viruses. Mice $(n=5)$ were infected intranasally with serial 10-fold dilutions of each virus, from $10^{4}$ to $10^{1} \mathrm{PFU}$ per mouse. The mock animals (circles) were inoculated with PBS/BSA. Following infection, mice were weighed daily, and the average body weights \pm s.d. of surviving animals in each group up to day 14 post infection are indicated as percentages of the original body weights. Dashed line indicates $75 \%$ of initial body weight. (f-i) Survival of mice after infection with $\mathrm{rSH} / 1$ (squares) or $\mathrm{rSH} / 1: \mathrm{AH} / 1-\mathrm{NA}$ (triangles) viruses or mock treated (circles). Kaplan-Meier curves represent survival after infection with each virus at 10 -fold dilutions from $10^{4}$ to $10^{1} \mathrm{PFU}$ or mock infection with PBS/BSA ( $n=5$ per group). Please note that experiments $\mathbf{b}-\mathbf{e}$ and $\mathbf{f}-\mathbf{i}$ share the same control mock-infected animals. Error bars represent s.d. in all panels. 
specifically attributed to oseltamivir-resistant quasi-species. Importantly, we show here that a purely oseltamivir-resistant virus clone of Shanghai/1 is as virulent in mice and as transmissible in guinea pigs as one encoding the oseltamivirsensitive Anhui/1-NA.

The majority of published studies on H7N9 isolates to date have used Anhui/1 as a representative virus ${ }^{25,27-30}$. In our experiments, we compared the replicative, virulence and transmission phenotypes of the oseltamivir-sensitive Anhui/1NA to the oseltamivir-resistant Shanghai/1-NA by expressing both NA genes in identical viral backbones. It should be noted that the Shanghai/1 and Anhui/1 NAs differ by two amino acids, the resistance-conferring catalytic residue $\mathrm{R} 292 \mathrm{~K}$, as well as the $\mathrm{N}$-terminal residue G39S (N9, G40S ${ }^{11}$ ) in the membraneproximal NA stalk. Certain NA stalk substitutions and deletions are known to affect NA sialidase activity; though most that have been studied are found in the membrane-distal stalk region ${ }^{31-33}$, our data cannot exclude the possibility that the G39S substitution exerts some effect on Shanghai/1-NA activity or viral fitness.

Nevertheless, in the PR/8 background, the oseltamivir-resistant Shanghai/1-NA demonstrated an $\sim 80 \%$ decrement in enzymatic activity and affinity for the fluorogenic substrate MUNANA, similar to impairments seen in N2 NAs encoding $R 292 K^{23}$. Surprisingly, though, in H7N9 viruses, oseltamivir resistance had no demonstrable effect on viral replication or pathogenicity in

\begin{tabular}{|c|c|c|c|}
\hline \multirow[t]{2}{*}{$\begin{array}{l}\text { Virus dose (PFU per } \\
\text { mouse) }\end{array}$} & \multicolumn{2}{|c|}{$\begin{array}{l}\text { Median time to death (days) } \\
\text { (number of survivors) }\end{array}$} & \multirow[t]{2}{*}{$P$-value } \\
\hline & $\begin{array}{c}\text { rSH/1:AH/1-NA } \\
\text { (R292) }\end{array}$ & $\begin{array}{c}\mathrm{rSH} / 1 \\
\text { (R292K) }\end{array}$ & \\
\hline $10^{4}$ & $6(0 / 5)$ & $6(0 / 5)$ & 0.649 \\
\hline $10^{3}$ & $7(0 / 5)$ & $6(0 / 5)$ & $0.0495^{\star}$ \\
\hline $10^{2}$ & $9(1 / 5)$ & $8(0 / 5)$ & $0.0442^{\star}$ \\
\hline $10^{1}$ & $>14(5 / 5)$ & $>14(5 / 5)$ & 1.000 \\
\hline
\end{tabular}

mice or on transmissibility among guinea pigs. These results stand in stark contrast to oseltamivir-resistant, NA-292Kencoding influenza $\mathrm{A}(\mathrm{H} 3 \mathrm{~N} 2)$ viruses, which have demonstrated marked reductions in murine virulence and ferret transmission ${ }^{17,19,22,34}$. This discrepancy is most likely due to the different genetic backgrounds in which these NA subtypes are expressed. Even among influenza viruses of the same subtype, antiviral resistance mutations can result in strikingly different replication, virulence and transmissibility phenotypes. For example, in the $\mathrm{N} 1$ subtype, the NA-H274Y substitution (N2 numbering) is the most commonly found oseltamivir resistance mutation, and it has been reported to compromise the in vitro and in vivo fitness of older seasonal H1N1 strains like A/Texas/ 36/1991 and A/New Caledonia/20/1999 (refs 35-37). However, it has also been shown to have minimal or no effect on viral fitness in the 2009 pandemic H1N1 background ${ }^{21,38-40}$, and even to enhance the transmissibility of A/Brisbane/59/2007-like viruses in the guinea pig model ${ }^{14}$. Thus, the genetic background of human H1N1 strains appears to determine whether the NA-H274Y oseltamivir resistance mutation is harmful, neutral or beneficial to virus fitness. Our data cannot conclusively predict whether the Shanghai/1-NA, encoded in the gene constellation of a different H7N9 virus such as Anhui/1 or Shanghai/2, would facilitate or hinder virulence or transmission of the resulting reassortant. However, our experiments do suggest that a fitness penalty may not always accompany the NA-R292K substitution in emergent H7N9 viruses.

Fitness in influenza A viruses is a multigenic trait. Our results clearly indicate that decreased NA enzymatic activity and substrate affinity in vitro do not invariably portend decreased viral replicative ability, virulence or transmissibility in vivo. We hypothesize that mutations in the NA gene, particularly in the enzymatic active site, alter the context of viral gene expression overall, perhaps compensating for reduced sialidase activity. In pre-2009 H1N1 viruses, for example, the NA-H274Y oseltamivir resistance mutation was shown to decrease surface expression of $\mathrm{NA}^{13}$. When the NA content of our 7:1 PR/8:N9 reassortant virus stocks was equivalent, the $\mathrm{PR} / 8$ :AH/1-NA reassortant had 2-4 times more receptor-binding activity than $\mathrm{PR} / 8: \mathrm{SH} / 1-\mathrm{NA}$, as measured by haemagglutination activity (Table 2). Relatively
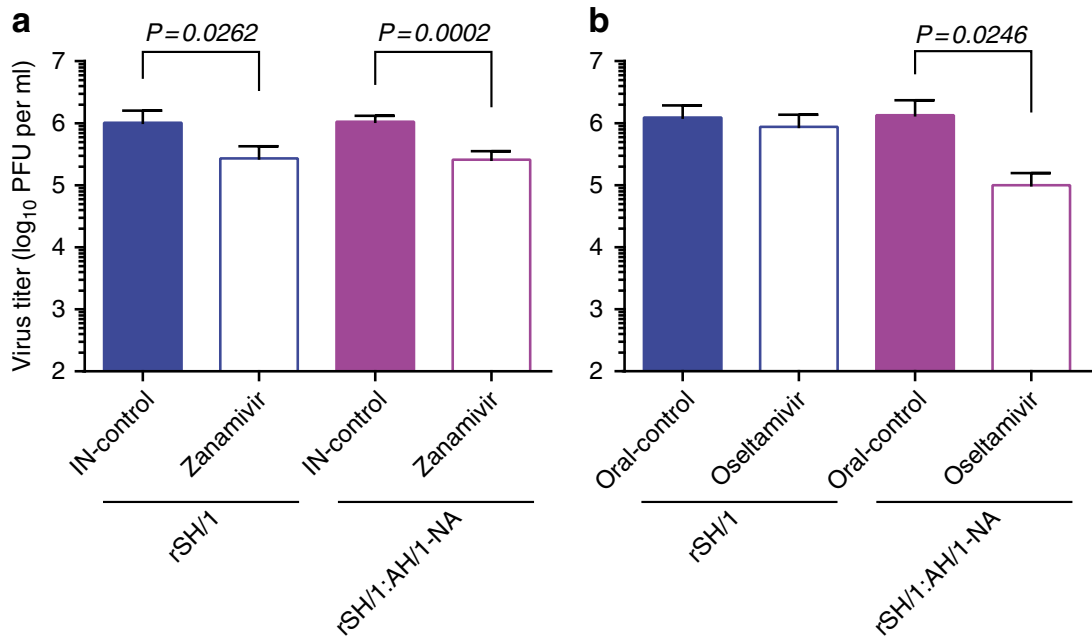

Figure 3 | Oseltamivir does not suppress H7N9 virus replication in mice infected with rSH/1. Female BALB/c mice (6-8-week-old; $n=5)$ were infected intranasally with $100 \mathrm{PFU}$ of $\mathrm{rSH} / 1$ (blue) or $\mathrm{rSH} / 1: \mathrm{AH} / 1-\mathrm{NA}$ (purple) viruses. Mice were treated with intranasal zanamivir (a), oral oseltamivir (b), intranasal PBS vehicle (a) or oral distilled water vehicle (b) twice daily for 5 days. Five mice were euthanized at day 5 post infection and viral lung titres were determined by plaque assays in MDCK cells. Student's $t$-test was applied to evaluate the statistical significance between viral titres of control animals and antiviral-treated animals. Error bars indicate s.d. value. 
Table 4 | Airborne respiratory transmission of rSH/1 and rSH/1:AH/1-NA in guinea pigs.

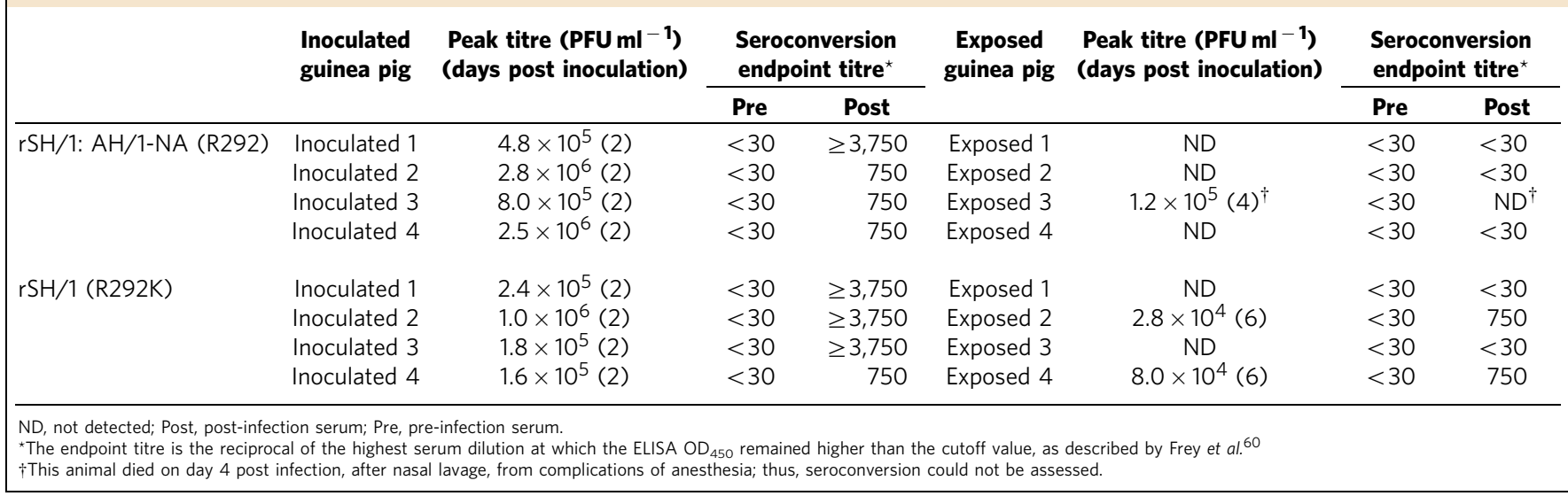

more PR/8 HA may be incorporated into virions when it is paired with the AH/1-NA than with the SH/1-NA. The fivefold decrease in activity and affinity of the $\mathrm{SH} / 1-\mathrm{NA}$, relative to that of the $\mathrm{AH} /$ $1-\mathrm{NA}$, could be offset by a roughly threefold decrease in surface HA activity, resulting in indistinguishable measures of viral fitness in vitro and in vivo. While our experiments cannot yet confirm this hypothesis, they do bring to light a finding of great clinical concern: a novel influenza A virus, isolated from an infected human, was able to develop high-level oseltamivir resistance without demonstrable loss of replicative ability in vitro or of virulence or transmissibility in vivo.

The prudent use of NA inhibitors in H7N9 influenza disease will be critical. In our experiments, we have shown that, at clinically relevant doses, orally administered oseltamivir does not suppress replication of the oseltamivir-resistant $\mathrm{rSH} / 1$ virus in the mouse lung. Oseltamivir demonstrates concentration-dependent pharmacodynamics, meaning that high peak drug concentrations at the site of infection are critical to its efficacy ${ }^{41}$. However, serum oseltamivir levels can be adversely affected by poor absorption from the gastrointestinal tract or impaired first-pass metabolism of the oral pro-drug ${ }^{42}$. World Health Organization (WHO) and US Centers for Disease Control and Prevention (CDC) guidelines have suggested that double-dose oral oseltamivir therapy or, where available, parenteral NA inhibitors, may be considered for severe avian influenza disease $e^{4,43,44}$. According to current data, intravenous zanamivir appears to maintain the best efficacy against the NA-R292K mutation ${ }^{24-26}$ while achieving reliably high peak plasma concentrations ${ }^{45}$. However, where parenteral NA inhibitors are not yet approved for use, clinicians treating suspected or confirmed H7N9 influenza virus infection may consider initiating oseltamivir treatment at double or triple doses until an alternative agent can be obtained, given the apparent ease with which the NA-R292K mutation is selected under oseltamivir therapy ${ }^{11,12}$ and the safety and tolerability profile of oseltamivir ${ }^{46,47}$. A recent randomized controlled trial failed to find clinical benefit with double-dose oseltamivir in severe influenza ${ }^{48}$; however, the rationale for high-dose oseltamivir in H7N9 infection is to lessen the risk of selecting for resistant variants during treatment. Clearly, however, pre-clinical and clinical trials are needed to provide definitive guidance. Given the potentially severe hazards to human health posed by oseltamivirresistant influenza A viruses, ongoing surveillance for the NA-R292K substitution will be critical, particularly in NA inhibitor-treated persons with H7N9 influenza disease.

In summary, even though we observed significant differences in the in vitro enzymatic activity of oseltamivir-sensitive and -resistant N9 NAs, we found that the oseltamivir-resistant NA affected neither H7N9 viral replication in primary human respiratory tract cells nor morbidity or mortality in mice, compared with an isogenic virus expressing a similar oseltamivir-sensitive NA. Airborne transmission of our recombinant viruses among guinea pigs was relatively inefficient, as has generally been seen in ferrets inoculated with the Anhui/1, Shanghai/1 or Shanghai/2 clinical isolates ${ }^{25,27-30,49}$. However, of note, we observed no significant difference in transmissibility between viruses encoding the oseltamivir-sensitive Anhui/1-NA or the oseltamivir-resistant Shanghai/1-NA. Thus, unlike viruses of the H3N2 subtype, acquisition of oseltamivir resistance in the emergent H7N9 strain is accompanied by no measurable fitness cost in any of the models in which our experiments were performed.

\section{Methods}

Cells. 293T and MDCK cells were obtained from the American Type Culture Collection (ATCC) and were maintained either in Dulbecco's minimal essential medium (DMEM) or in minimal essential medium (MEM; Gibco) supplemented with $10 \%$ fetal calf serum (HyClone) and penicillin-streptomycin (Gibco), respectively. Recombinant viruses were grown in 10-day-old specific-pathogen-free embryonated hen's eggs (Charles River Laboratories) at $37^{\circ} \mathrm{C}$ for 2 days. Human tracheobronchial epithelial cells (hTBE; Lonza) were differentiated by seeding them on $12-\mathrm{mm}$ Transwell filters (pore size, $0.4 \mu \mathrm{m}$; Corning) that were coated with collagen type I from human placenta (Sigma-Aldrich) in 12-well plates (Corning). Cells were incubated with a 1:1 mixture of bronchial epithelial cell growth medium (BEGM; Lonza) and DMEM. When cells became confluent, liquid from the upper compartment was removed and cells were differentiated in an air-liquid interface for 4-6 weeks. Medium in the lower compartment was renewed every $48 \mathrm{~h}^{50}$.

Construction of plasmids. All eight viral genes of the influenza A/Shanghai/1/ 2013(H7N9) strain (SH/1; GISAID accession numbers EPI439486-EPI439494) were in vitro-synthesized by GeneArt Gene Synthesis (Invitrogen). Since the $5^{\prime}$ and $3^{\prime}$ noncoding regions of the SH/1 viral RNA segments had not been reported, the genes were constructed with noncoding regions corresponding to the consensus sequences of closely related influenza A viruses. The NA gene of influenza A/Anhui/1/2013(H7N9) (AH/1; EPI439509) was kindly supplied by Dr Richard Webby, St Jude Children's Hospital, Memphis, TN, USA. The viral gene segments were then subcloned into the ambisense expression vector $\mathrm{pDZ}^{51}$.

Rescue of recombinant chimeric influenza $A$ viruses. Five recombinant influenza viruses-wild-type Shanghai/1 (rSH/1), a 7:1 reassortant between Shanghai/1 and the NA gene of Anhui/1 (rSH/1:AH/1-NA); 7:1 reassortants of A/Puerto Rico/ 8/34(H1N1) (PR/8) encoding the NA genes of either Shanghai/1 (rPR/8:SH/1-NA) or Anuhi/1 (rPR/8:AH/1-NA) and $\mathrm{rPR} / 8: \mathrm{SH} / 1-\mathrm{HA} / \mathrm{NA}$, a 6:2 reassortant between the PR/8 internal genes and the Shanghai/1-HA and -NA genes-were rescued from plasmid DNA 52,53 . The eight $\mathrm{pDZ}$ plasmids encoding the viral gene segments (1 $\mu \mathrm{g}$ each) were co-transfected into $293 \mathrm{~T}$ cells with Lipofectamine 2000

(Invitrogen), according to the manufacturer's protocol. At $24 \mathrm{~h}$ post-transfection, the virus-containing cell supernatant was inoculated by 29 -gauge syringe into the 
allantoic cavities of 10-day-old embryonated chicken eggs, which were incubated at $37^{\circ} \mathrm{C}$ for 2 days and then slowly cooled overnight at $4{ }^{\circ} \mathrm{C}$. Allantoic fluid was collected by aseptically opening the allantoic cavity through the air sac and aspirating the allantoic fluid by pipette. The presence of virus in allantoic fluid was evaluated by haemagglutination of chicken red blood cells, and virus stock titres were determined by plaque assay of 10 -fold dilutions of allantoic fluid in PBS on MDCK cells overlaid with $0.65 \%$ agar (Oxoid Ltd.) in MEM supplemented with $0.4 \%$ BSA, penicillin-streptomycin, $0.01 \%$ DEAE dextran and $1 \mu \mathrm{g} \mathrm{ml}^{-1}$ tosylsulfonyl phenylalanyl chloromethyl ketone (TPCK)-trypsin ${ }^{54}$.

In vitro enzyme assays. Reassortants of $\mathrm{PR} / 8$ encoding either the Shanghai/1-NA (rPR/8:SH/1-NA) or the Anhui/1-NA (rPR/8:AH/1-NA) were used for all in vitro assays at biosafety level (BSL)-2. For both Michaelis-Menten and NA inhibitor resistance assays, reactions were performed in $33 \mathrm{mM} 2$-(N-morpholino) ethanesulfonic acid (MES; Sigma-Aldrich) with $4 \mathrm{mM}$ calcium chloride at $\mathrm{pH}$ 6.6 (MES buffer), with a fluorogenic NA substrate, $2^{\prime}$-(4-methylumbelliferyl)- $\alpha$-D-N-acetylneuraminic acid (MUNANA; Sigma-Aldrich), in 96-well plates (Corning) ${ }^{14,55}$. Relative fluorescence units (RFU) produced by cleaved MUNANA were measured with the Synergy H1 Hybrid Multi-Mode Microplate Reader and Gen5 software (BioTek).

Determination of Michaelis-Menten parameters for H7N9 NAs. NA content of stock 7:1 reassortant viruses $\mathrm{rPR} / 8: \mathrm{SH} / 1-\mathrm{NA}$ and $\mathrm{rPR} / 8: \mathrm{AH} / 1-\mathrm{NA}$ were quantified by western blot of serial virus dilutions, using antisera raised in mice against an influenza $\mathrm{A}(\mathrm{H} 11 \mathrm{~N} 9)$ virus as a primary antibody. Band densitometry was performed with ImageJ (NIH), and viruses were normalized to equivalent NA content by dilution in MES buffer. In 96-well plates, the normalized viruses were added to 1.67-fold dilutions of MUNANA substrate (11 concentrations ranging from 6-1,000 $\mu \mathrm{M})$ and a twelfth control well of MES buffer without MUNANA to measure background fluorescence. Plates were incubated at $37^{\circ} \mathrm{C}$ in the microplate reader, and the Gen 5 software recorded the RFUs produced by catalysed MUNANA substrate every $90 \mathrm{~s}$ for $40 \mathrm{~min}$, at excitation and emission wavelengths of 360 and $448 \mathrm{~nm}$, respectively. For each MUNANA concentration, the measured RFUs (minus background fluorescence) were plotted against time to calculate reaction velocity (in $\mathrm{RFU} \mathrm{s}^{-1}$ ) by linear regression. Michaelis-Menten parameters $K_{\mathrm{m}}$ and $V_{\max }$ were determined by non-linear regression of the MUNANA concentration versus velocity plots in Prism 6 (GraphPad Software $)^{14}$. Two independent experiments, with three replicates per experiment, were performed, and results are expressed as an average of all replicates \pm s.e.

Determination of H7N9 resistance to NA inhibitors. Serial dilutions of stock viruses $\mathrm{rPR} / 8: \mathrm{SH} / 1-\mathrm{NA}$ and $\mathrm{rPR} / 8: \mathrm{AH} / 1-\mathrm{NA}$ were incubated in a saturating concentration of MUNANA $(400 \mu \mathrm{M})$ for $1 \mathrm{~h}$ at $37^{\circ} \mathrm{C}$ to obtain the $50 \%$ effective virus concentration $\left(\mathrm{EC}_{50}\right)$, at which half the substrate was catalysed. Viruses were then diluted to the $\mathrm{EC}_{50}$ in MES buffer for use in a fluorometric neuraminidase inhibition assay) ${ }^{14,55}$. Three NA inhibitors, in 10-fold dilutions, were evaluated: oseltamivir carboxylate (Toronto Research Chemicals, North York, ON: $1 \mathrm{mM}-1 \mathrm{pM}$ ), peramivir (MedChemexpress, Princeton, NJ; $1 \mathrm{mM}-1 \mathrm{pM}$ ), or zanamivir (GlaxoSmithKline, Philadelphia, PA; $0.1 \mathrm{mM}-0.1 \mathrm{pM}$ ). In 96-well plates, normalized viruses were added to 10 drug dilutions plus 2 no-drug control wells to measure uninhibited NA activity. For each drug, the plate also contained a row of no-virus control wells, including the 10 drug dilutions mixed only with MES buffer, to measure background fluorescence. Plates were incubated at room temperature for $45 \mathrm{~min}$, and then MUNANA $(400 \mu \mathrm{M})$ was added to all wells, followed by a 1-h incubation at $37^{\circ} \mathrm{C}$. Substrate conversion was stopped by the addition of $140 \mathrm{mM}$ sodium hydroxide in $83 \%$ ethanol, and RFUs of catalysed MUNANA substrate were measured at excitation and emission wavelengths of 360 and $448 \mathrm{~nm}$ on a microplate reader. After subtracting the background fluorescence (as measured in the no-virus control wells), the NA activity at each drug concentration was expressed as a percentage of uninhibited NA activity by dividing the RFUs produced at each of the 10 drug concentrations by the average of the RFUs produced in the two no-drug control wells. The $\mathrm{IC}_{50}$ of each drug for the $\mathrm{SH} / 1$ and $\mathrm{AH} / 1$ NAs was determined by plotting the percent of uninhibited NA activity as a function of the $\log _{10}$ of the drug concentration, and the data were fit to a doseresponse curve by non-linear regression in Prism 6. A minimum of six replicates per virus/drug pair were performed, and results are expressed as an average of all replicates with a $95 \%$ confidence interval $(95 \% \mathrm{CI})$.

Growth kinetics of recombinant viruses in hTBE cells. Differentiated hTBE cells were infected at an MOI of 0.01 in PBS with $0.3 \%$ BSA in an air-liquid interface. Cells were subsequently cultured with BEGM in the lower compartment and incubated at either 33 or $37^{\circ} \mathrm{C}$. Viral supernatants were collected by adding $100 \mu \mathrm{l}$ of PBS with $0.3 \%$ BSA to each well (surface area: $100 \mathrm{~mm}^{2}$ ). Cells were incubated for $30 \mathrm{~min}$, viral supernatants were collected and viral titres determined by plaque assay on MDCK cells.

Animals. All research studies involving the use of animals were reviewed and approved by the Institutional Animal Care and Use Committees (IACUC) of the
Icahn School of Medicine at Mount Sinai and Rutgers, the State University of New Jersey. This study was carried out in strict accordance with the recommendations in the Guide for the Care and Use of Laboratory Animals of the National Research Council (8th Edition).

Female BALB/c mice (6-8-week-old) were purchased from Jackson Laboratories (Bar Harbor, ME). All viral infections of mice were performed in accordance with CDC and USDA guidelines in the Animal Biosafety Level (ABSL)-3 + facility at the Icahn School of Medicine at Mount Sinai. Procedures were performed in accordance with Animal Welfare Act guidelines and approved by the Mount Sinai Institutional Animal Care and Use and Institutional Biosafety Committees. Briefly, five groups of mice ( $n=5$ per group) were anesthetized by intraperitoneal injection of a mixture of ketamine $\left(100 \mathrm{mg} \mathrm{kg}^{-1}\right)$ and xylazine $\left(5 \mathrm{mg} \mathrm{kg}^{-1}\right)$ and infected intranasally with one of four virus doses $\left(10^{1}, 10^{2}, 10^{3}\right.$ or $\left.10^{4} \mathrm{PFU}\right)$ in $50 \mu \mathrm{l}$ of PBS with $0.3 \%$ BSA or mock-infected with $50 \mu \mathrm{l}$ of PBS with $0.3 \%$ BSA. The mice were monitored daily for clinical signs of illness and for weight loss. Upon reaching $75 \%$ of initial body weight, animals were humanely euthanized by a lethal dose of ketamine and xylazine ${ }^{56}$. Kaplan-Meier survival curves were plotted in Prism 6 and compared by the log-rank test. The median mouse lethal dose $\left(\mathrm{mLD}_{50}\right)$ was calculated according to the method of Reed and Muench ${ }^{57}$, with $95 \%$ CI determined by the method of Thakur and Fezio ${ }^{58}$. Viral lung titres were determined by standard plaque assay on MDCK cells.

Six-week-old female Hartley strain guinea pigs, weighing 400-450 g, were obtained from Charles River Laboratories (Montréal, QC). All viral infections of guinea pigs were performed in accordance with CDC and USDA guidelines in the ABSL-3 facility at the Regional Biocontainment Laboratory at Rutgers, the State University of New Jersey. Procedures were performed in accordance with Animal Welfare Act guidelines and approved by the Rutgers Institutional Animal Care and Use and Institutional Biosafety Committees. Guinea pigs were anesthetized by intramuscular injection of a mixture of ketamine $\left(30 \mathrm{mg} \mathrm{kg}^{-1}\right)$ and xylazine $\left(5 \mathrm{mg} \mathrm{kg}^{-1}\right)$ prior to phlebotomy, virus inoculation, nasal lavage and humane euthanasia by $\mathrm{CO} 2$ inhalation followed by bilateral thoracotomy ${ }^{56}$.

Virus susceptibility to NA inhibitors in vivo. Female BALB/c mice (6-8-weekold) were infected intranasally with $100 \mathrm{PFU}$ of either $\mathrm{rSH} / 1$ or rSH/1:AH/1-NA in a total volume of $50 \mu \mathrm{l}$. Starting $1 \mathrm{~h}$ post infection, groups of five mice were administered one of four preparations: (1) intranasal zanamivir (GlaxoSmithKline), $25 \mathrm{mg} \mathrm{kg}^{-1}$ in $50 \mu \mathrm{l}$ of PBS, twice daily for 5 days; (2) oral oseltamivir phosphate (Roche), $50 \mathrm{mg} \mathrm{kg}^{-1}$ in $400 \mu \mathrm{l}$ of distilled water, twice daily for 5 days; (3) intranasal PBS, $50 \mu \mathrm{l}$ twice daily for 5 days; or (4) distilled water orally, $400 \mu \mathrm{l}$ twice daily for 5 days. Mice were euthanized on day 5 post infection, and virus titres in lung tissues were determined by plaque assay on MDCK cells.

Airborne respiratory transmission experiments. Guinea pig transmission experiments were performed with $\mathrm{rSH} / 1$ and $\mathrm{rSH} / 1: \mathrm{AH} / 1-\mathrm{NA}$, with ABSL-3 containment, in the Regional Biocontainment Laboratory at Rutgers, the State University of New Jersey. During the experiment, ambient temperatures in the facility ranged from $67-72^{\circ} \mathrm{F}\left(19-22^{\circ} \mathrm{C}\right)$, with $42-48 \%$ relative humidity. Each virus was assessed in four pairs of guinea pigs, with one virus-inoculated and one virus-exposed animal per pair. On day 0 , an inoculum of $10^{4} \mathrm{PFU}$ of either rSH/1 or $\mathrm{rSH} / 1: \mathrm{AH} / 1-\mathrm{NA}$ in $300 \mu \mathrm{l}$ was administered intranasally to four animals per group, and infected guinea pigs were then sequestered from naive animals to prevent cross-contamination. At day 1 post-inoculation, each guinea pig was individually housed in a modified $31 \times 31 \times 19 \mathrm{~cm}$ polycarbonate cage (\#4, Thoren Caging Systems, Hazleton, PA), from one side of which a $20 \times 10 \mathrm{~cm}$ opening had been cut and replaced with a panel of $1.25-\mathrm{cm}$ wire mesh. The cages of one virusinoculated and one virus-exposed guinea pig were aligned in pairs so that the wire mesh sides were directly opposed, allowing air to flow freely between cages but precluding direct contact between animals. Each inoculated-exposed guinea pig pair's cages were placed in an isolator cabinet in a negative-pressure containment unit (Model RVB243116US6; Allentown, Inc., Allentown, NJ). On days 2, 4, 6 and 8 post infection, nasal lavages were performed by instilling a total of $1 \mathrm{ml}$ of PBS into the nostrils and allowing it to drain by gravity onto a sterile Petri dish. Samples were collected into 1.5 -ml tubes, centrifuged to pellet debris, and stored at $-80^{\circ} \mathrm{C}$. Virus in nasal lavage specimens were titrated by plaque assay of 10 -fold dilutions of thawed lavage fluid in PBS on MDCK cells, as described above ${ }^{20}$.

Seroconversion was assessed by ELISA of pre- and post-infection serum samples from guinea pigs. In 96-well ELISA plates (4 HBX Immunoassay Plates, Immulon), $50 \mu \mathrm{l}$ of purified $6: 2$ reassortant virus $\mathrm{rPR} / 8: \mathrm{SH} / 1-\mathrm{HA} / \mathrm{NA}$, at a concentration of $2 \mu \mathrm{g} \mathrm{ml}^{-1}$ in PBS, was applied to coat the well bottoms during an overnight incubation at $4{ }^{\circ} \mathrm{C}$. Virus was removed, and plates were blocked with $5 \%$ non-fat milk in PBS for $1 \mathrm{~h}$ at room temperature. Plates were washed with PBS, and guinea pig sera (in fivefold dilutions between $1 / 30$ and 1/3,750, in duplicate) were added to the plate and incubated for $1 \mathrm{~h}$ at room temperature. Plates were washed again with PBS and then incubated with $50 \mu \mathrm{l}$ of a 1:2,500 dilution of alkaline phosphatase-linked anti-guinea pig secondary antibody (Abcam) for $1 \mathrm{~h}$ at room temperature. Plates were washed again with PBS, and $50 \mu \mathrm{l}$ of $p$-nitrophenyl phosphate substrate (Invitrogen) was then added. After $30 \mathrm{~min}$, the reaction was stopped with $50 \mu \mathrm{l}$ of $0.75 \mathrm{~N} \mathrm{NaOH}$, and the plates were read at $405 \mathrm{~nm}$ in an ELISA plate reader (DTX 880 multimode detector; Beckman Coulter) ${ }^{59}$. The ELISA positivity cutoff value was determined from the pre-infection serum 
samples, using a $99.9 \%$ upper confidence limit, according to the method of Frey et al. ${ }^{60}$, and the endpoint titre was defined as the reciprocal of the highest serum dilution with $\mathrm{OD}_{450}$ above the cutoff value.

Transmission events were determined to have occurred in exposed guinea pigs with infectious virus isolated from nasal lavage specimens by plaque assay and with H7N9-specific antibodies detected in serum by ELISA. Transmission efficiencies were calculated as the number of exposed guinea pigs infected by transmission from an inoculated partner animal, divided by the total number exposed, and were compared by two-tailed Fisher's exact test in Prism 6.

\section{References}

1. De Jong, M. D. et al. Oseltamivir resistance during treatment of influenza A (H5N1) infection. N. Engl. J. Med. 353, 2667-2672 (2005).

2. European Centre for Disease Prevention and Control (ECDC). ECDC/WHO frequently asked questions for Oseltamivir Resistance http://ecdc.europa.eu/en/ healthtopics/seasonal_influenza/antivirals/pages/influenza_antiviral_resistance oseltamivir.aspx (2008).

3. Le, Q. M. et al. Avian flu: isolation of drug-resistant H5N1 virus. Nature 437, 1108 (2005).

4. World Health Organization (WHO). Overview of the emergence and characteristics of the avian influenza A(H7N9) virus http://www.who.int/ influenza/human_animal_interface/influenza_h7n9/WHO_H7N9_review_ 31May13.pdf (2013).

5. World Health Organization (WHO). Influenza at the human-animal interface: Summary and assessment as of 4 June $2013 \mathrm{http} / / /$ www.who.int/influenza/ human_animal_interface/Influenza_Summary_IRA_HA_;interface_04Jun 13.pdf; (2013).

6. Borse, R. H. et al. Effects of vaccine program against pandemic influenza A(H1N1) virus, United States, 2009-2010. Emerg. Infect. Dis. 19, 439-448 (2013).

7. Hsu, J. et al. Antivirals for treatment of influenza: a systematic review and meta-analysis of observational studies. Ann. Intern. Med. 156, 512-524 (2012).

8. De Vries, E. et al. Influenza A virus entry into cells lacking sialylated N-glycans. Proc. Natl Acad. Sci. USA 109, 7457-7462 (2012).

9. Varghese, J. N., Epa, V. C. \& Colman, P. M. Three-dimensional structure of the complex of 4-guanidino-Neu5Ac2en and influenza virus neuraminidase. Protein Sci. 4, 1081-1087 (1995).

10. McKimm-Breschkin, J. L. Influenza neuraminidase inhibitors: antiviral action and mechanisms of resistance. Influenza Other Respir Viruses 7(Suppl 1): 25-36 (2013).

11. Gao, R. et al. Human infection with a novel avian-origin influenza A (H7N9) virus. N. Engl. J. Med. 368, 1888-1897 (2013).

12. Hu, Y. et al. Association between adverse clinical outcome in human disease caused by novel influenza A H7N9 virus and sustained viral shedding and emergence of antiviral resistance. Lancet 381, 2273-2279 (2013).

13. Bloom, J. D., Gong, L. I. \& Baltimore, D. Permissive secondary mutations enable the evolution of influenza oseltamivir resistance. Science 328, 1272-1275 (2010).

14. Bouvier, N. M., Rahmat, S. \& Pica, N. Enhanced mammalian transmissibility of seasonal influenza $\mathrm{A} / \mathrm{H} 1 \mathrm{~N} 1$ viruses encoding an oseltamivir-resistant neuraminidase. J. Virol. 86, 7268-7279 (2012).

15. Seibert, C. W., Rahmat, S., Krammer, F., Palese, P. \& Bouvier, N. M. Efficient transmission of pandemic H1N1 influenza viruses with high-level oseltamivir resistance. J. Virol. 86, 5386-5389 (2012).

16. Abed, Y., Baz, M. \& Boivin, G. Impact of neuraminidase mutations conferring influenza resistance to neuraminidase inhibitors in the $\mathrm{N} 1$ and $\mathrm{N} 2$ genetic backgrounds. Antivir. Ther. 11, 971-976 (2006).

17. Gubareva, L. V., Robinson, M. J., Bethell, R. C. \& Webster, R. G. Catalytic and framework mutations in the neuraminidase active site of influenza viruses that are resistant to 4-guanidino-Neu5Ac2en. J. Virol. 71, 3385-3390 (1997).

18. McKimm-Breschkin, J. L. et al. Mutations in a conserved residue in the influenza virus neuraminidase active site decreases sensitivity to Neu5Ac2enderived inhibitors. J. Virol. 72, 2456-2462 (1998).

19. Yen, H. L. et al. Neuraminidase inhibitor-resistant influenza viruses may differ substantially in fitness and transmissibility. Antimicrob. Agents Chemother. 49, 4075-4084 (2005).

20. Bouvier, N. M., Lowen, A. C. \& Palese, P. Oseltamivir-resistant influenza A viruses are transmitted efficiently among guinea pigs by direct contact but not by aerosol. J. Virol. 82, 10052-10058 (2008).

21. Seibert, C. W. et al. Oseltamivir-resistant variants of the 2009 pandemic H1N1 influenza A virus are not attenuated in the guinea pig and ferret transmission models. J. Virol. 84, 11219-11226 (2010).

22. Carr, J. et al. Influenza virus carrying neuraminidase with reduced sensitivity to oseltamivir carboxylate has altered properties in vitro and is compromised for infectivity and replicative ability in vivo. Antiviral. Res. 54, 79-88 (2002).

23. Zurcher, T. et al. Mutations conferring zanamivir resistance in human influenza virus $\mathrm{N} 2$ neuraminidases compromise virus fitness and are not stably maintained in vitro. J. Antimicrob. 58, 723-732 (2006).
24. Sleeman, K. et al. R292K Substitution and Drug Susceptibility of Influenza A(H7N9) Viruses. Emerg. Infect. Dis. 19, 1521-1524 (2013).

25. Watanabe, T. et al. Characterization of H7N9 influenza A viruses isolated from humans. Nature 501, 551-555 (2013).

26. Yen, H. L. et al. Resistance to neuraminidase inhibitors conferred by an R292K mutation in a human influenza virus $\mathrm{H} 7 \mathrm{~N} 9$ isolate can be masked by a mixed R/K viral population. MBio 4, e00396-00313 (2013).

27. Belser, J. A. et al. Pathogenesis and transmission of avian influenza A (H7N9) virus in ferrets and mice. Nature 501, 556-559 (2013).

28. Zhang, Q. et al. H7N9 influenza viruses are transmissible in ferrets by respiratory droplet. Science 341, 410-414 (2013).

29. Richard, M. et al. Limited airborne transmission of H7N9 influenza A virus between ferrets. Nature 501, 560-563 (2013).

30. Xu, L. et al. Novel avian-origin human influenza $\mathrm{A}(\mathrm{H} 7 \mathrm{~N} 9)$ can be transmitted between ferrets via respiratory droplets. J. Infect. Dis. doi:10.1093/infdis/jit474 (2013).

31. Castrucci, M. R. \& Kawaoka, Y. Biologic importance of neuraminidase stalk length in influenza A virus. J. Virol. 67, 759-764 (1993).

32. Matsuoka, Y. et al. Neuraminidase stalk length and additional glycosylation of the hemagglutinin influence the virulence of influenza H5N1 viruses for mice. J. Virol. 83, 4704-4708 (2009).

33. Yano, T., Nobusawa, E., Nagy, A., Nakajima, S. \& Nakajima, K. Effects of singlepoint amino acid substitutions on the structure and function neuraminidase proteins in influenza A virus. Microbiol. Immunol. 52, 216-223 (2008).

34. Tai, C. Y. et al. Characterization of human influenza virus variants selected in vitro in the presence of the neuraminidase inhibitor GS 4071. Antimicrob. Agents Chemother. 42, 3234-3241 (1998).

35. Baz, M., Abed, Y., Simon, P., Hamelin, M. E. \& Boivin, G. Effect of the neuraminidase mutation $\mathrm{H} 274 \mathrm{Y}$ conferring resistance to oseltamivir on the replicative capacity and virulence of old and recent human influenza $\mathrm{A}(\mathrm{H} 1 \mathrm{~N} 1)$ viruses. J. Infect. Dis. 201, 740-745 (2010).

36. Herlocher, M. L. et al. Influenza viruses resistant to the antiviral drug oseltamivir: transmission studies in ferrets. J. Infect. Dis. 190, 1627-1630 (2004).

37. Ives, J. A. et al. The H274Y mutation in the influenza A/H1N1 neuraminidase active site following oseltamivir phosphate treatment leave virus severely compromised both in vitro and in vivo. Antiviral Res. 55, 307-317 (2002).

38. Hamelin, M. E. et al. Oseltamivir-resistant pandemic A/H1N1 virus is as virulent as its wild-type counterpart in mice and ferrets. PLoS Pathog. 6, e1001015 (2010).

39. Kiso, M. et al. Characterization of oseltamivir-resistant 2009 H1N1 pandemic influenza A viruses. PLoS Pathog. 6, e1001079 (2010).

40. Memoli, M. J. et al. Multidrug-resistant 2009 pandemic influenza A(H1N1) viruses maintain fitness and transmissibility in ferrets. J. Infect. Dis. 203, 348-357 (2011).

41. Rayner, C. R. et al. Pharmacokinetic-pharmacodynamic determinants of oseltamivir efficacy using data from phase 2 inoculation studies. Antimicrob. Agents Chemother. 57, 3478-3487 (2013).

42. Wattanagoon, Y. et al. Pharmacokinetics of high-dose oseltamivir in healthy volunteers. Antimicrob. Agents Chemother. 53, 945-952 (2009).

43. Abdel-Ghafar, A. N. et al. Update on avian influenza A (H5N1) virus infection in humans. N. Engl. J. Med. 358, 261-273 (2008).

44. Centers for Disease Control and Prevention (CDC). Interim Guidance on the Use of Antiviral Agents for Treatment of Human Infections with Avian Influenza A (H7N9) http://www.cdc.gov/flu/avianflu/h7n9-antiviraltreatment.htm (2013).

45. Cass, L. M., Efthymiopoulos, C. \& Bye, A. Pharmacokinetics of zanamivir after intravenous, oral, inhaled or intranasal administration to healthy volunteers. Clin. Pharmacokinet. 36(Suppl 1): 1-11 (1999).

46. Nicholson, K. G. et al. Efficacy and safety of oseltamivir in treatment of acute influenza: a randomised controlled trial. Neuraminidase Inhibitor Flu Treatment Investigator Group. Lancet 355, 1845-1850 (2000).

47. Treanor, J. J. et al. Efficacy and safety of the oral neuraminidase inhibitor oseltamivir in treating acute influenza: a randomized controlled trial. US Oral Neuraminidase Study Group. JAMA 283, 1016-1024 (2000).

48. South East Asia Infectious Disease Clinical Research Network. Effect of double dose oseltamivir on clinical and virological outcomes in children and adults admitted to hospital with severe influenza: double blind randomised controlled trial. BMJ 346, f3039 (2013).

49. Zhu, H. et al. Infectivity, transmission, and pathology of human-isolated H7N9 influenza virus in ferrets and pigs. Science 341, 183-186 (2013).

50. Ramos, I. et al. Effects of receptor binding specificity of avian influenza virus on the human innate immune response. J. Virol. 85, 4421-4431 (2011).

51. Quinlivan, M. et al. Attenuation of equine influenza viruses through truncations of the NS1 protein. J. Virol. 79, 8431-8439 (2005).

52. Fodor, E. et al. Rescue of influenza A virus from recombinant DNA. J. Virol. 73, 9679-9682 (1999).

53. Neumann, G. et al. Generation of influenza A viruses entirely from cloned cDNAs. Proc. Natl Acad. Sci. USA 96, 9345-9350 (1999). 
54. Gaush, C. R. \& Smith, T. F. Replication and plaque assay of influenza virus in an established line of canine kidney cells. Appl. Microbiol. 16, 588-594 (1968).

55. Hurt, A. Fluorometric neuraminidase inhibition assay: Standard operating procedure WHO-025 (WHO Collaborating Centre for Reference \& Research on Influenza, Australia, http://www.nisn.org/documents/A.Hurt_Protocol_for_ NA_fluorescence.pdf, 2007).

56. Panel on Euthanasia. AVMA Guidelines for the Euthanasia of Animals 2013 (Schaumburg, IL: American Veterinary Medical Association, 2013)

57. Reed, L. J. \& Muench, H. A simple method of estimating fifty per cent endpoints. Am. J. Hyg. 27, 493-497 (1938).

58. Thakur, A. K. \& Fezio, W. L. A computer program for estimating LD50 and its confidence limits using modified Behrens-Reed-Muench cumulant method. Drug Chem. Toxicol. 4, 297-305 (1981).

59. Pica, N., Chou, Y. Y., Bouvier, N. M. \& Palese, P. Transmission of influenza B viruses in the guinea pig. J. Virol. 86, 4279-4287 (2012).

60. Frey, A., Di Canzio, J. \& Zurakowski, D. A statistically defined endpoint titer determination method for immunoassays. J. Immunol. Methods 221, 35-41 (1998).

\section{Acknowledgements}

We are grateful to David Perlin and Steven Park of the Regional Biocontainment Laboratory at Rutgers, the State University of New Jersey, for making the facility available for the guinea pig transmission experiments and enabling their completion. We thank Richard Webby for providing the cDNA corresponding to the NA gene of the influenza A/Anhui/1/2013(H7N9) virus, and Richard Cadagan and Osman Lizardo for excellent technical assistance. This work was supported by the Center for Research in Influenza Pathogenesis (CRIP), a Center of Excellence in Influenza Research and Surveillance (CEIRS) funded by the NIH/NIAID (contract \# HHSN266200700010C). F.K. is supported by an Erwin Schrödinger fellowship (J 3232) from the Austrian Science Fund (FWF). N.M.B. was supported by an NIH/NIAID Career Development Grant (K08 AI089940).

\section{Author contributions}

R.H., M.S., A.G.-S., P.P. and N.M.B. conceived and designed the experiments. R.H., M.S. V.H.L.-G., R.R.T., I.M., E.L.J., A.S. and N.M.B. performed the experiments and analysed the data. R.H., M.S. and N.M.B. wrote the manuscript. V.H.L.-G., I.M., F.K., A.S., A.G.-S and P.P. edited the manuscript.

\section{Additional information}

Competing financial interests. The authors declare no competing financial interests.

Reprints and permissions information is available online at http://npg.nature.com/ reprintsandpermissions/.

How to cite this article: Hai, R. et al. Influenza A(H7N9) virus gains neuraminidase inhibitor resistance without loss of in vivo virulence or transmissibility. Nat. Commun. 4:2854 doi: 10.1038/ncomms3854 (2013).

This work is licensed under a Creative Commons AttributionNonCommercial-NoDerivs 3.0 Unported License. To view a copy of this license, visit http://creativecommons.org/licenses/by-nc-nd/3.0/ 